\title{
Microbiologie de la lèpre
}

\begin{abstract}
Mycobacterium leprae, agent de la lèpre, se distingue des autres mycobactéries par la faible teneur de son génome en bases $G$ et $\mathbf{C}$ et par une structure particulière de son peptidoglycane. Il coexiste dans les lésions lépreuses avec certaines corynébactéries qui pourraient jouer un rôle adjuvant dans la pathogénicité de l'agent infectieux. Les différents antigènes et immunomodulateurs des parois microbiennes, qui jouent un rôle essentiel dans les différentes phases de la maladie lépreuse, commencent seulement à être connus, notamment grâce à l'emploi d'anticorps monoclonaux et à l'utilisation des techniques des recombinants d'ADN.
\end{abstract}

\section{Carlo Cocito}

Docteur en médecine et en chimie Professeur à l'université de Louvain

\section{Marc Coene \\ Docteur ès sciences \\ Chercheur qualifié}

\section{Jean Delville}

Docteur en médecine

Professeur émérite à l'université de Louvain

\section{ADRESSE}

C. Cocito, M. Coene, J. Delville : unité de microbiologie générale et de génétique moléculaire, ICP, université de Louvain, école de médecine, avenue Hippocrate 75, B-1200, Bruxelles, Belgique. èpre et tuberculose sont parmi les maladies infectieuses les plus anciennes et les plus répandues : quarante-cinq millions de malades chroniques, sources d'infection pour cent millions de personnes par an.

\section{Aspects cliniques et immunologiques}

La lèpre est une mycobactériose systémique, qui affecte tous les tissus du corps, bien que les lésions les plus apparentes intéressent la peau et les nerfs. Les malades qui, à certains stades de la lèpre, éliminent des quantités considérables de bacilles par la voie des muqueuses, sont la source principale de contagion. Toutefois, la découverte récente de bactéries identiques au bacille lépreux chez les tatous [1], les singes [2] et dans certains lieux privilégiés, a fait envisager la possibilité d'une transmission de la maladie autre que celle entre les humains. Dans la maladie lépreuse, on distingue : un pôle tuberculoïde (la forme hyper-réactive ou TT) et un pôle lépromateux (le stade anergique ou LL), séparés par des formes intermédiaires (ou borderline, $\mathrm{BT}, \mathrm{BB}$ et $\mathrm{BL}$ ). D'habitude, la maladie débute par une forme indéterminée évoluant parfois en stade $\mathrm{TT}$, où la barrière immunologique bloque l'infection en dressant une immunité antilépreuse. Chez une minorité des patients, toutefois, l'affection évolue graduellement du stade TT vers les formes borderline, qui parfois progressent vers le pôle lépromateux, irréversible.

Le granulome tuberculoïde renferme des cellules géantes de Langhans, des cellules épithélioïdes, des histiocytes, des cellules lymphoïdes et des plasmocytes. Les macrophages spongieux et bourrés de bacilles (cellules de Virchow) représentent la population dominante du granulome lépromateux. Dans la lèpre tuberculoïde, les granulomes immunologiques des nerfs périphériques (neurotropisme caractéristique de Mycobacterium leprae) causent une dégénérescence des fibres nerveu- 


\section{RÉFÉRENCES}

1. Portaels F, Pattyn SR. Isolation of fastidiously growing mycobacteria from armadillo infected with Mycobacterium leprae. Int J Lepr 1982 ; 50 : 370-4.

2. Meyers WM, Binford $\mathrm{CH}$, Walsh GP, et al. Animal models of leprosy. In : Leive L, Schlessinger D, eds. Microbiology 1984. Washington : American Soc of Microbiol, 1984 : 307-11.

3. Mehra V, Brennan PJ, Rada E, Convit J, Bloom BR. Lymphocyte suppression in leprosy induced by unique $M$. leprae glycolipid. Nature $1984 ; 308$ : 194-6.

4. Cocito C, Delville J. Properties of microorganisms from human leprosy lesions. A review. Rev Infect Dis 1983 ; 5 : 649-57.

5. Cocito C, Delville J. Biological, chemical, immunological, and staining properties of bacteria isolated from tissues of leprosy patients. Eur J Epidemiol 1985; 1 : 202-31.

6. Brown S, Lanéelle M, Asselineau J, Barksdale L. Description of Conynebacterium tuberculostearicum sp. nov., a leprosy derived corynebacterium. Ann Microbiol (Paris) 1984 ; 135 : 251-67.

\section{*ABRÉVIATIONS*}

CMN : groupe de micro-organismes incluant les genres Corynebacterium + Rhodococcus, Mycobacterium et Nocardia.

MAIS : mycobactéries des sousgroupes M. avium, M. intracellulare et M. scrofulaceum, agents étiologiques de mycobactérioses non tuberculeuses (" tuberculose atypique" ").

LDC " "leprosy-derived corynebacteria"): groupe de corynébactéries issues de lésions lépreuses. Nouvelle appellation : Corynebacterium tuberculostearicum $s p$. nov [6]. $A D M$ (" armadillo-derived mycobacteria "): groupe de mycobactéries isolées de tatous sauvages ou injectés par des lépromes humains.

BCG : bacille de Calmette-Guérin, souche atténuée de Mycobacterium bovis.

TMA (" thermostable macromolecular antigens") : famille d'antigènes majeurs des organismes CMN incluant l'A60 de M. bovis, l'A7 de M. leprae et le $M_{1}$ des $L D C$. mDAP : méso-diamino-pimélate (acide aminé inusité). ses, qui produit des paralysies, des altérations sensorielles et une détérioration du trophisme cutané. Les stades tuberculoïdes (TT) et borderline (BT) sont caractérisés par de violentes réactions immunitaires. En revanche, l'anergie immunologique lépromateuse (qui ressemble au phénomène de tolérance immunitaire, à la seule exception que des anticorps sont ici produits) est caractérisée par une inactivité des phagocytes visà-vis du bacille de Hansen. Cela est dû essentiellement à l'incapacité des lymphocytes $\mathrm{T}$ à activer les macrophages, qui phagocytent donc les bacilles lépreux sans pouvoir les détruire. La production d'un glycolipide phénolique à propriété immunosuppressive [3], ainsi que la synthèse réduite d'interleukine 2, semblent impliquées dans ce phénomène de tolérance.

Le diagnostic de la lèpre est basé sur l'identification de l'agent étiologique dans les lésions périphériques multibacillaires et sur l'image histologique. Les tests cutanés d'hypersensibilité retardée aident à établir le pronostic de la maladie. A l'inoculation intradermique de lépromine (homogénat phéniqué* de tissu lépromateux), font suite soit une infiltration de cellules mononucléées du derme dans les cas hyper-réactifs (TT, $\mathrm{BT}, \mathrm{BB})$, soit une réaction négative dans les cas d'anergie (BL et LL). La réaction est évaluée après un ou deux jours (test de Fernandez) et après un mois (test de Mitsuda) : elle révèle la présence d'une immunité cellulaire, dont les phénomènes d'hypersensibilité retardée sont l'expression.

\section{Les micro-organismes}

Mycobacterium leprae a été mis en évidence dans un granulome lépromateux par G.H. Armauer Hansen à Bergen en 1873. Le procédé de référence pour l'identification du bacille de Hansen a

\footnotetext{
* Homogénal phéniqué : c'est un broyat de granulome lépromateux chauffé, auquel on ajoule 0,5\% de phénol.
}

été pendant presque un siècle celui de Ziehl-Neelsen, qui utilise le déparaffinage des coupes au xylène et la coloration à la carbolfuchsine (acido-résistance): $M$. leprae apparaît sous la forme de bâtonnets rouges, parfois assemblés dans des amas (globi) (figure 1, p. 467). Sous l'influence d'un traitement antimicrobien, $M$. leprae peut perdre son acido-résistance au Ziehl-Neelsen sur des coupes histologiques classiques, alors que, sur des frottis ou sur des coupes à congélation, l'acido-résistance est conservée. On peut y remédier et restaurer l'acido-résistance en soumettant les coupes histologiques classiques enrobées dans la paraffine à diverses colorations spéciales** (Tableau I). Le Gram permet également la coloration de $M$. leprae issu de sujets traités, mais cette technique n'est pas spécifique de cette mycobactérie (figure 1) [4, 5]. Une corrélation entre l'homogénéité de coloration et la multiplication in vivo de la bactérie a été établie, les organismes granuleux correspondant in vivo aux bactéries incapables de se multiplier.

La découverte de microorganismes accessoires (c'est-à-dire ne s'identifiant pas à $M$. leprae) dans les lésions lépreuses est attribuée à Bordoni-Uffreduzzi (1888). Ce ne fut qu'environ $3 / 4$ de siècle plus tard que ces mêmes bactéries furent redécouvertes indépendamment dans les laboratoires de L. Barksdale (New York university), J. Delville (université de Louvain) et C. Reich (Philippines) $[4,6]$. Ces micro-organismes furent appelés "diphtéroïdes " à cause de leur ressemblance avec Corynebacterium diphteriae, l'agent étiologique de la diphtérie humaine (figure 1). Contrairement à $M$. leprae, dont la multiplication en culture axénique*** n'a pas jusqu'à présent été obtenue, la propagation in vitro des diphtéroïdes est obtenue dans un pourcen-

\footnotetext{
* Colorations de Fite-Faraco, Nyka, Wade-Fite et à l'auramine, après déparaffinage selon Wade.

*** Culture axénique : culture microbienne en milieu nutritionnel défini, sans cellules hötes.
} 
tage élevé de cas [5]. La ressemblance morphologique des diphtéroïdes isolés de patients de tous les pays, de même que leur présence si fréquente dans les lésions de lèpre et leur absence dans la peau normale, suggèrent une relation directe entre ces bactéries et la lèpre. Par ailleurs, ces mêmes organismes ont été isolés d'animaux (souris, tatous) inoculés expérimentalement par des homogénats de lépromes humains.

L'impossibilité de multiplier $M$. leprae in vitro a été un obstacle majeur à la recherche sur la lèpre. Des progrès dans ce domaine ont été réalisés à la suite de la découverte de certains systèmes de multiplication de cet organisme in vivo. L'inoculation de $M$. leprae dans le capiton plantaire de la souris est suivie d'une multiplication limitée des bactéries atteignant en six mois un plateau situé 2-3 logarithmes au-dessus du point de départ $\left(10^{3}-10^{4}\right)$. Une prolifération plus prolongée a lieu chez la souris athymique (jusqu'à $10^{10}$ bacilles dans la patte, lieu d'injection) et dans certains tissus ou organes [7]. L'injection d'homogénats de lépromes chez le tatou à neuf bandes (Dasypus novemcinctus) produit dans un certain nombre de cas une maladie généralisée avec multiplication bactérienne dans tous les organes et mort de l'animal après environ deux ans [8]. Les quantités importantes de mycobactéries obtenues à partir d'homogénats de foie, de

\begin{tabular}{|c|c|c|c|c|c|c|c|}
\hline \multicolumn{8}{|c|}{$\begin{array}{c}\text { Tableau } 1 \\
\text { TECHNIQUES DE COLORATION DES MYCOBACTÉRIES }\end{array}$} \\
\hline \multirow{2}{*}{$\begin{array}{c}\text { Dénomination } \\
\begin{array}{c}\text { (auteur/réactif } \\
\text { de base) }\end{array}\end{array}$} & \multicolumn{2}{|c|}{ Matériel d'analyse } & \multirow{2}{*}{$\begin{array}{l}\text { Déparaffinage } \\
\text { des coupes }\end{array}$} & \multirow[t]{2}{*}{ Coloration } & \multirow[t]{2}{*}{ Décoloration } & \multicolumn{2}{|c|}{ Évaluation * } \\
\hline & Frottis & $\begin{array}{l}\text { Coupes } \\
\text { histo- } \\
\text { logiques }\end{array}$ & & & & Frottis & Coupes \\
\hline \multirow[t]{2}{*}{ Ziehl-Neelsen } & + & & & $\begin{array}{l}\text { fuchsine } \\
\text { phéniquée }\end{array}$ & alcool-acide & 2 & \\
\hline & & + & toluène & $\begin{array}{l}\text { fuchsine } \\
\text { phéniquée }\end{array}$ & alcool-acide & & 7 \\
\hline Wade-Fite & & + & $\begin{array}{l}\text { thérébentine-huile } \\
\text { de paraffine }\end{array}$ & $\begin{array}{l}\text { néofuchsine } \\
\text { phéniquée }\end{array}$ & $\begin{array}{l}\text { formol-acide + } \\
\text { permanganate- } \\
\text { oxalate }\end{array}$ & & 1 \\
\hline Fite-Faraco & & + & $\begin{array}{l}\text { xylène-huile vé- } \\
\text { gétale }\end{array}$ & $\begin{array}{l}\text { fuchsine } \\
\text { phéniquée }\end{array}$ & alcool-acide & & 3 \\
\hline \multirow[t]{3}{*}{ Nyka } & + & & & $\begin{array}{l}\text { acide périodique } \\
\text { fuchsine phéni- } \\
\text { quée }\end{array}$ & alcool-acide & 3 & \\
\hline & & + & $\begin{array}{l}\text { thérébentine-huile } \\
\text { de paraffine }\end{array}$ & $\begin{array}{l}\text { fuchsine } \\
\text { phéniquée }\end{array}$ & alcool-acide & & 3 \\
\hline & & + & toluène & $\begin{array}{l}\text { acide périodique } \\
\text { fuchsine phéni- } \\
\text { quée }\end{array}$ & alcool-acide & & 4 \\
\hline \multirow[t]{2}{*}{ Auramine } & + & & & $\begin{array}{l}\text { auramine phéni- } \\
\text { quée }\end{array}$ & alcool-acide & 1 & \\
\hline & & + & $\begin{array}{l}\text { thérébentine-huile } \\
\text { de paraffine }\end{array}$ & $\begin{array}{l}\text { auramine } \\
\text { phéniquée }\end{array}$ & alcool-acide & & 1 \\
\hline \multirow[t]{2}{*}{ Gram } & + & & & cristal violet & alcool-acétone & 3 & \\
\hline & & + & toluène & $\begin{array}{l}\text { cristal violet } \\
\text { phéniqué }\end{array}$ & alcool-acétone & & 3 \\
\hline
\end{tabular}

Colorations de contraste : bleu de méthylène ou vert de malachite pour les frottis, Carazzi ou trichrome Masson, ou bleu Evans pour les coupes.

* Évaluation des techniques par ordre décroissant de performance 11 = la méthode la plus performante dans toutes les conditions, 7 = méthode insuffisante en cas de chimiothérapie) et de spécificité. 


\section{RÉFÉRENCES}

7. Shepard CC. The experimental disease that follows the injection of human leprosy bacilli into footpads of mice. $J$ Exp Med $1960 ; 112$ : 445-54.

8. Kirchheimer WF, Storrs EE. Attempts to establish the armadillo (Dasypus novemcinctus Linn) as a model for the study of leprosy. I. Report of lepromatoid leprosy in experimentally infected armadillo. Int J Lepr $1971 ; 39$ : 693-702.

9. Galletti G, Cavicchi G, Ussia G. Replication of Mycobacterium leprae in hibernating ground squirrels (Citellus tridecemlineatus). Acta Leprol (Genève) 1982 ; 88 : 23-31.

10. Mukherjee R, Mahadevan PR, Antia NH. Organized nerve culture. I. A technique to study the effect of $M$. leprae infection. Int $J$ Lepr $1980 ; 48$ : 183-8.

11. Imaeda I, Kirchheimer WF, Barksdale L. DNA isolated from Mycobacterium leprae: genome size, base ratio, and homology with other related bacteria as determined by optical DNA-DNA reassociation. J Bacteriol 1982 ; 150: 414-7.

12. Danhaive $\mathrm{P}$, Hoet $\mathrm{P}$, Cocito $\mathrm{C}$. Composition of the DNA of diphtheroid microorganisms and other corynebacteria. Int J System Bact 1982; 32 : 70-6.

13. Draper P. Cell walls of Mycobacterium leprae. Int J Lepr 1976 ; 44 : 95-8.

14. Janczura E, Leyh-Bouille M, Ghuysen JM, Cocito C. Structure of the peptidoglycans obtained from a group of Corynebacteria. J Bacteriol $1981 ; 145$ : 775-9.

15. Abou-Zeid C, Voiland A, Michel G, Cocito G. Structure of the wall polysaccharide isolated from a group of corynebacteria. Eur J Biochem 1982 ; 128 : 363-70.

16. Gailly C, Sandra P, Verzele M, Cocito C. Analysis of mycolic acids from a group of corynebacteria by capillary gas chromatography and mass spectrometry. Eur $J$ Biochern 1982 ; 125 : 83-94.

17. Draper P, Dobson G, Minnikin DE, Minnikin SM. The mycolic acids of Mycobacterium leprae harvested from experimentally infected nine-banded armadillos. Ann Microbiol (Paris) rate et de peau d'animaux infectés ont servi, notamment, aux recherches récentes sur $M$. leprae ainsi qu'à la préparation du vaccin OMS (Organisation Mondiale de la Santé). Le processus d'hibernation chez certains écureuils (Citellus tridecemlineatus) est accompagné d'une involution du système immunitaire : l'inoculation de $M$. leprae à ce moment précis est suivie de sa multiplication, qui s'arrête au printemps et reprend l'hiver suivant, entraînant ainsi la mort du rongeur [9]. On a également décrit un système in vitro intéressant. La mise en culture de ganglions nerveux de souris est accompagnée d'une prolifération de cellules de Schwann. Le bacille de Hansen pénètre dans ces cellules par phagocytose et s'y multiplie [10].

On a pu mettre en évidence, grâce à l'emploi expérimental des tatous, des bactéries assimilables à $M$. leprae (et donc non cultivables) et des mycobactéries non répertoriées se multipliant très lentement in vitro. Ces dernières ont été appelées ADM (armadillo-derived mycobacteria) et classées en cinq sous-groupes (ADM I à V), sur la base de leurs propriétés phénotypiques (composition en acides mycoliques et exigences nutritionnelles) [1]. La spécificité et les rôles des ADM sont inconnus.

\section{Les génomes}

Le classement des différentes espèces de mycobactéries entre elles, au sein du genre Mycobacterium, et par rapport aux genres proches (genres Corynebacterium, Rhodococcus, Nocardia), c'est-à-dire dans le groupe CMN, peut être réalisé grâce à l'étude de leur ADN. Un intérêt supplémentaire de telles études est de pouvoir se servir de certaines souches plus faciles à cultiver et taxonomiquement proches, soit comme modèles pour des espèces pathogènes plus exigeantes, soit comme sources d'antigènes, voire comme vaccins. Les trois paramètres fondamentaux concernant l'ADN microbien sont : (a) la composition en bases ; (b) la susceptibilité aux enzymes de restriction; (c) l'homologie avec les génomes des bactéries apparentées.

$\mathrm{La}$ composition en bases de l'ADN est habituellement établie par la séparation et l'évaluation des bases puriques (A et $G$ ) et pyrimidiques ( $\mathrm{C}$ et $\mathrm{T}$ ) obtenues par hydrolyse, procéde laborieux qui requiert une quantité importante d'ADN. En réalité, puisque A s'apparie seulement avec $\mathrm{T}$ et $G$ avec $C$, il suffit d'évaluer le pourcentage en $\mathrm{G}+\mathrm{C}(\% \mathrm{GC})$, par la mesure soit de la densité de flottaison, soit de la température moyenne de fusion $\left(\mathrm{T}_{\mathrm{m}}\right)$ de l'ADN*. Une relation mathématique relie, en fait, ces propriétés physiques de l'ADN à sa composition chimique. Un procédé très récent d'analyse enzymatique consiste à copier la matrice d'ADN par l'ADN polymérase, en employant deux précurseurs radioactifs (un pour la paire AT et l'autre pour GC) : le rapport entre les deux marqueurs incorporés permet d'évaluer le \% GC à partir de nanogrammes d'ADN. Par ce procédé, l'ADN de $M$. leprae a été analysé et son \% GC s'est révélé être égal à 55,5 (F. Hottat et al., résultats en voie de publication). La mesure du $\mathrm{T}$ avait donné une valeur proche de $56 \%$ [11]. Or, il faut savoir que les génomes des bactéries du groupe CMN ont des valeurs de \% GC allant de 50 à 60 pour les corynébactéries, de 60 à 70 pour les rhodocoques, de 62 à 70 pour les mycobactéries et de 67 à 70 pour les nocardias. Dès lors, $M$. leprae devrait être exclu du genre Mycobacterium, tenant compte de la composition en nucléotides de son génome.

L'étude du génome des diphtéroïdes a mis en évidence un fait surprenant : le \% GC de toutes les souches de ce groupe se rapproche d'une même valeur de $56 \%$ [12]. Ces données montrent l'homogénéité génétique de ce groupe de bactéries et leur appar-

\footnotetext{
* Les régions riches en $G C$ sont plus denses que les régions riches en $A T$. De plus, la liaison $G=C$ est plus stable que la liaison $A=T$ et la température nécessaire pour la dissocier est donc supérieure.
} 
tenance au genre Corynebacterium (50-60 \% GC) : d'où leur appellation de leprosy-derived corynebacteria (LDC). En revanche, l'analyse des génomes des souches appartenant au groupe ADM a fourni des valeurs en \% GC allant de 62 à 68 (M. de Kesel et al., résultats en voie de publication). Ces données témoignent de l'hétérogénéité génétique de ce groupe d'organismes, et confirment en même temps leur appartenance au genre Mycobacterium.

Toutefois, les renseignements fournis par la composition en bases de l'ADN portent exclusivement sur une parenté lointaine. C'est le test d'hybridation qui donne une mesure de la similitude existant dans les séquences de deux $\mathrm{ADN}$. Ce test consiste à chauffer un mélange de deux ADN (l'un inconnu, l'autre de référence) au-delà de la température de fusion. Les deux chaînes de la double hélice se séparent, à la suite de la cassure des ponts hydrogène, et, lors de l'abaissement progressif de la température, on assiste à la formation d'une double hélice hybride renfermant les deux brins antiparallèles des deux ADN : le pourcentage d'ADN bicaténaire ainsi formé donne la mesure du degré d'homologie entre les deux génomes. De telles études ont exclu qu'il y ait un degré élevé d'homologie entre les différents types de bactéries associées à la lèpre $(\mathrm{M}$. De Kesel et al., résultats en voie de publication). D'autre part, un haut niveau d'homologie entre différentes souches de LDC a été enregistré, confirmant ainsi l'homogénéité génétique du groupe. Enfin, un faible niveau d'homologie a été retrouvé lors de l'hybridation génomique entre différents sous-groupes d'ADM, alors que l'homologie entre souches appartenant à un même sous-groupe était élevée. Le groupe ADM peut par conséquent être assimilé à un genre (en l'occurrence, le genre Mycobacterium), les différents sous-groupes étant assimilables à des espèces, du point de vue taxonomique. Les enzymes de restriction (endo$\mathrm{m} / \mathrm{s} n^{\circ} 8$ vol. 3 , octobre 87

Tableau II

CLIVAGE DES GÉNOMES DES BACTÉRIES ASSOCIÉES A LA LÈPRE PAR LES ENDONUCLÉASES DE RESTRICTION

\begin{tabular}{|lc|ccc|}
\hline & Endonucléases & & ADN \\
\hline Symbole & Séquence reconnue & ML & ADM & LDC \\
\hline Dpn I & GAm TC & - & - & + \\
Sau 3A I & $\mathrm{GA}^{\circ} \mathrm{TC}^{-}$ & + & + & + \\
Mbo I & $\mathrm{GA}^{-} \mathrm{TC}^{\circ}$ & + & + & + \\
Taq I & $\mathrm{TC}^{\circ} \mathrm{GA}^{-}$ & + & & + \\
Hae III & $\mathrm{GGC}^{-} \mathrm{C}^{\circ}$ & + & + & + \\
Hpa II & $\mathrm{C}^{-} \mathrm{C}^{-} \mathrm{GG}$ & + & + & + \\
Alu I & $\mathrm{AGC}-\mathrm{T}^{-}$ & + & & + \\
\hline
\end{tabular}

Colonne de gauche : $m$ : clivage se faisant seulement en cas de base méthylée : - : clivage inhibé par la présence d'une base méthylée ; o : clivage ayant lieu indépendamment de la méthylation de bases.

Colonne de droite : $M L=$ Mycobacterium leprae $; A D M=$ armadillo-derived mycobacteria ; $L D C=$ leprosy-derived corynebacteria. Les signes + et - indiquent l'aptitude des endonucléases de restriction à cliver l'ADN.

nucléases de la classe II), qui clivent l'ADN au niveau de séquences de bases bien définies, se sont révélées des outils exceptionnels pour l'analyse de la structure fine du chromosome microbien. Certains groupes d'enzymes de restriction (isoschizomères) reconnaissent une même séquence différemment, selon qu'elle est méthylée ou non. C'est le cas, notamment, du groupe suivant d'isoschizomères qui inclut : Mbo I (qui reconnaît la séquence GATC dépourvue de bases méthylées), Dpn I (clivant cette même séquence seulement si son adénine est méthylée), et Sau 3AI (qui clive l'ADN dans les deux cas). L'emploi de ces isoschizomères a révélé que l'ADN des LDC était clivé par Dpn I et par Sau 3AI, mais pas par Mbo I, ce qui indiquait la présence d'adénine méthylée (MeA) dans la séquence GATC (F. Hottat $e t$ al., résultats en voie de publication). En revanche, les ADN de $M$. leprae et des souches d'ADM étaient coupés par Mbo
I et Sau 3AI, et non par Dpn I, montrant ainsi l'absence de méthylation au niveau de cette même séquence (Tableau II). Ces études ont donc révélé un marqueur génétique, $\mathrm{GA}^{\mathrm{m}} \mathrm{TC}$, spécifique des LDC, puisqu'il s'est révélé absent dans tous les autres micro-organismes du groupe CMN. De plus, nos travaux ont montré que, dans les LDC, toutes les séquences GATC présentes dans le génome, et seulement celles-ci, étaient méthylées et que ce même trait était commun à toutes les souches LDC (F. Hottat et al., résultats en voie de publication). Voici donc un nouvel élément qui démontre l'homogénéité et l'unicité de ce groupe de bactéries.

\section{Les parois}

Les composants de base des parois chez les micro-organismes du groupe CMN sont deux hauts polymères interconnectés, le peptidoglycane et le complexe 


\section{RÉFÉRENCES}

18. Lederer E, Adam A, Ciorbaru A, Petit JF, Wietzerbin J. Cell walls of mycobacteria and related organisms: chemistry and immuno-stimulant properties. Mol Cell Biochem $1975 ; 7: 87-104$.

19. Hunter SW, Fujiwara T, Brennan PJ Structure and antigenicity of the major specific glycolipid antigen of Mycobacterium leprae. $J$ Biol Chom 1982 ; 257 : 15072-8.

20. Cho SN, Fujiwara T, Hunter SW, Rea TH, Gelber RH, Brennan PJ. Use of an artificial antigen containing the 3,6-di-O-methylD-glucopyranosyl epitope for the serodiagnosis of leprosy. J Infect Dis 1984 ; 150 : 311-22.

21. Closs O, Mshana RN, Harboe M. Antigenic analysis of Mycobacterium leprae. Scand J Immunol $1979 ; 9$ : 297-302.

22. Cocito C, Vanlinden F. Preparation and properties of antigen 60 from Mycobacterium bovis BCG. Clin Exp Immunol 1986; 66 : 262-72.

23. Laub R, Delville J, Cocito C. Immunological relatedness of ribosomes from Mycobacteria, Nocardiae and Corynebacteria, and microorganisms in leprosy lesions. Infect Immun 1978 ; 22 : 540-7.

24. Gueur MC, Harboe M, Fontaine F, Delville J, Cocito $\mathrm{C}$. Comparison of the cytoplasmic antigens of leprosy-derived corynebacteria and some mycobacteria. Scand J Immunol 1983 ; 17 : 497-506.

25. Delville J, Spina A, Rajjan W, Cocito C. Modification of the kinetics of growth of Mycobacterium leprae by a group of corynebacteria. FEMS Microbiol Lett 1982 ; 15 : 253-6.

26. Ivanyi J, Sivha S, Aston $R$, Cussel D, Ken M, Sengupta U. Definition of speciesspecific and cross-reactive antigenic determinants of Mycobacterium leprae using monoclonal antibodies. Clin Exp Immunol 1983; 52 : 528-36.

27. Kolk AHJ, Ho ML, Klatser PR, el al. Production and characterization of monoclonal antibodies to Mycobacterium tuberculosis, $M$. bovis BCG and M. leprae. Clin Exp Immunol 1984 ; $58: 511-21$

28. Young RA, Mehra V, Sweetser D, et al. Genes for the major protein antigens of the leprosy parasite Mycobacterium leprae. Nature $1985 ; 316: 450-2$.

29. Young RA, Bloom BR, Grosskinsky CM, Ivanyi J, Thomas D, Davis RW. Dissection of Mycobacterium tuberculosis antigens using recombinant DNA. Proc Nall Acad Sci USA $1985 ; 82: 2583-7$.

30. Minnikin DE. The biology of the Mycobacteria, vol. 1. New York: Academic Press, 1982:
Figure 1. Morphologie des micro-organismes associés à la lèpre. Sections de granulomes lépreux colorées respectivement par les méthodes de ZiehlNeelsen (A) et Wade-Fite (B), la deuxième montrant un plus grand nombre de bactéries, et par un procédé de double coloration (Wade-Fite-hématoxyline) mettant en évidence deux types de bactéries (C). Une biopsie de lèpre lépromateuse traitée par le Ziehl (D), l'auramine (E), et les anticorps anti-LDC (leprosyderived corynebacterial fluorescents (F). Une section de nerf infiltré par $M$. leprae (G). Des cultures de macrophages humains infectées in vitro par LDC (H) et M. leprae (1). (D'après [4] et [5].) polysaccharide-mycolate (figure 2). Les extrémités des ramifications latérales du polysaccharide pariétal sont estérifiées aux acides mycoliques. Les longues chaînes hydrophobes de ces derniers forment saillie vers l'extérieur de la cellule (figure 2) : la considérable résistance présentée par ces organismes vis-à-vis des facteurs antimicrobiens est liée à cette épaisse couche hydrophobe. Ces mêmes composants de la paroi sont largement responsables des réactions immunitaires déclenchées chez l'hôte.

Le peptidoglycane comporte des chaînes linéaires de nature polysaccharidique auxquelles sont attachés des oligopeptides reliant les chaînes polyosiques entre elles (figure 3). L'analyse du peptidogly- cane de $M$. leprae a mis en évidence un tétrapeptide ne contenant pas de L-alanine, cet acide aminé étant complètement remplacé par la glycine [13] (aucun autre organisme du groupe CMN n'a de tel peptidoglycane). Le peptidoglycane des LDC possède un tétrapeptide typique des organismes CMN [14].

Le polysaccharide pariétal des mycobactéries est un arabinogalactane ramifié dont les extrémités sont estérifiées par les acides mycoliques. La structure du polysaccharide de la paroi de $M$. leprae est inconnue ; celui des LDC est un arabinogalactomannane (figure 3, p. 468) [15].

Les acides mycoliques sont des dérivés $\alpha$-alkyl $\beta$-hydroxy d'acides gras ayant des tailles et des com-

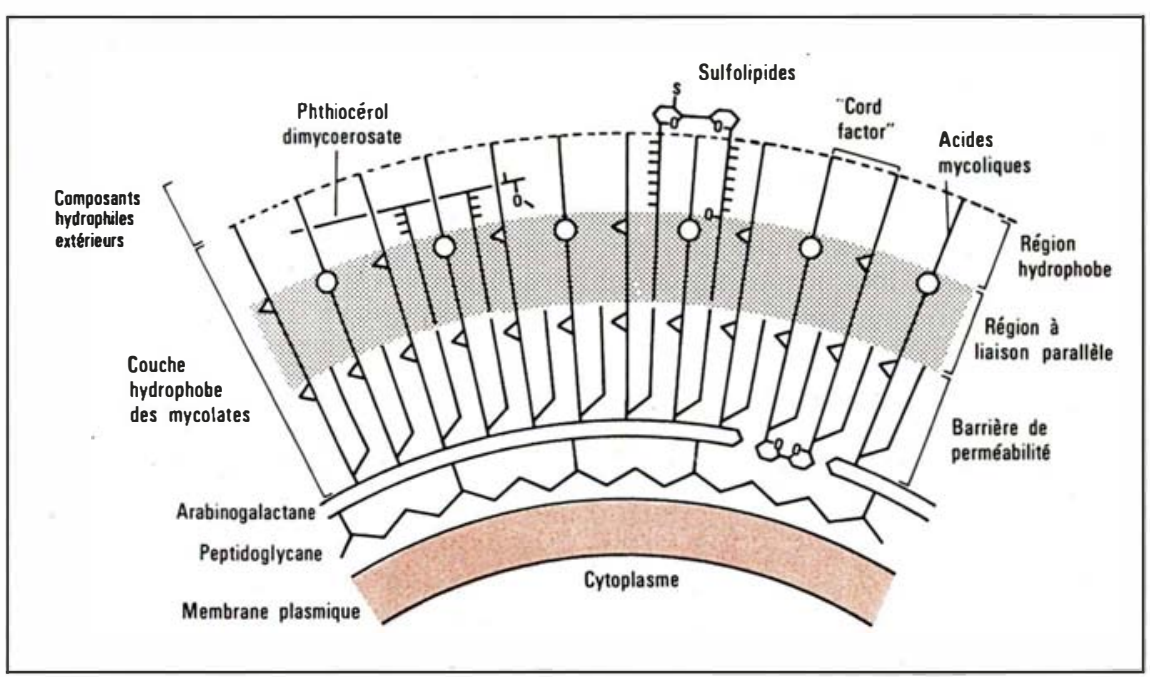

Figure 2. Schéma de la paroi mycobactérienne. Les deux polymères majeurs de la paroi, peptidoglycane et arabinogalactane-mycolate, entourant la membrane, sont schématisés ainsi que la couche hydrophobe extérieure formée de mycolates et cires. Des composants hydrosolubles (sulfolipides) recouvrent des parties de la couche hydrophobe extérieure. (D'après [30]). 


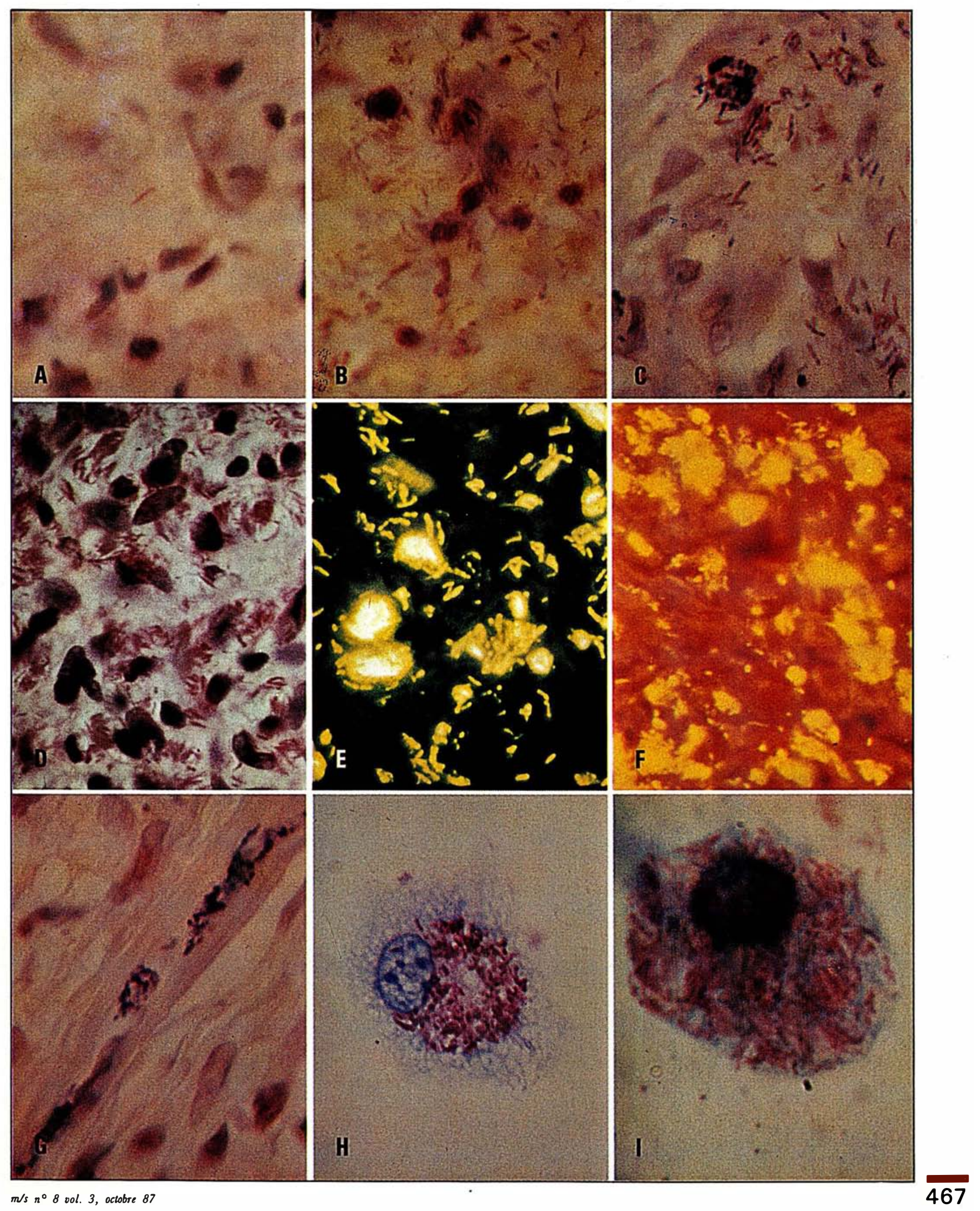




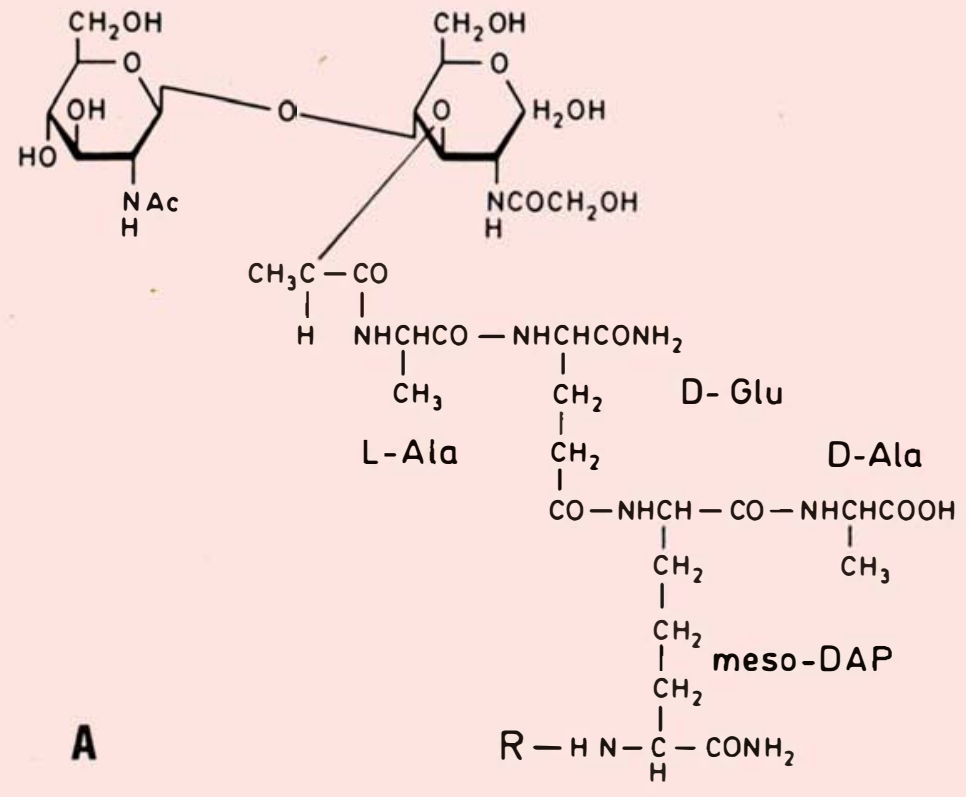

Figure 3. Structure des polymères pariétaux des LDC lleprosy-derived corynebacteria). A : le peptidoglycane des LDC (d'après [14] ); B : le polysaccharide pariétal des LDC (d'après [15]). meso-DAP = acide méso-diaminopimélique.

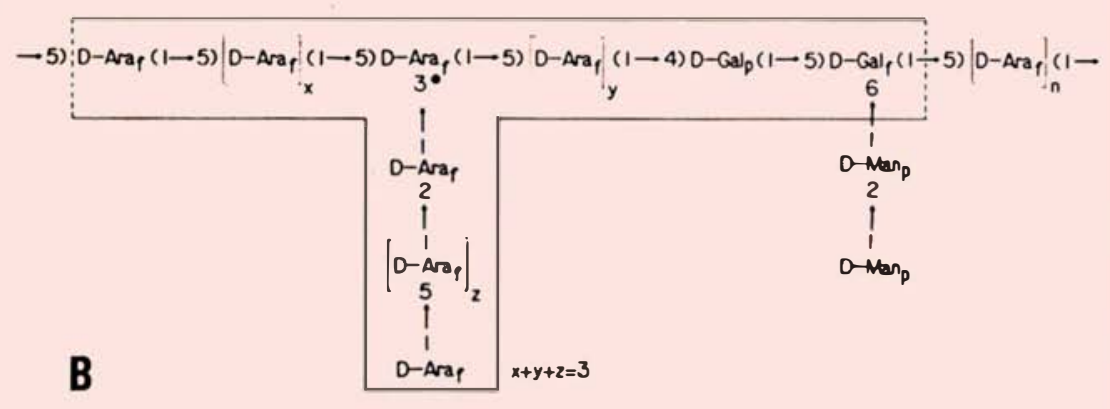

positions différentes chez les différents genres d'organismes $\mathrm{CMN}$, d'où leur intérêt taxonomique. Ainsi, les acides corynomycoliques possèdent 20 à 40 carbones, les nocardiques 36 à 66 , et les acides mycoliques mycobactériens 60 à 90 . Il suffit donc de fractionner par chromatographie en couche mince monodimensionnelle un lysat méthanolique bactérien pour en établir la position taxonomique. Toutes les

produisent une tache unique comigrante avec le standard d'acide corynomycolique (figure 4). $\mathrm{C}$ 'est suite à une pareille analyse que les diphtéroïdes ont été identifiés comme étant des vraies corynébactéries (d'où leur appellation LDC) [16]. A présent, la nouvelle appellation de Corynebacterium tuberculostearicum sp. nov. ${ }^{*}$ a été proposée, à cause de la présence, chez les LDC, d'acide tuberculostéarique [6]. En soumettant les extraits mycobactériens à la chro- matographie en couche mince bidimensionnelle, Draper et al. ont mis en évidence cinq images différentes : une de celles-ci était spécifique de $M$. leprae [17]. Les LDC possèdent des acides corynomycoliques de taille plus grande et plus insaturés que ceux de $C$. diphteriae [16]. Bien que les acides mycoliques issus des différents sous-groupes d'ADM soient tous de type mycobactérien, on peut reconnaître un dessin spécifique pour chaque sous-groupe [1].

\section{Les antigènes}

Les mycobactéries recèlent trois types de substances agissant sur le système immunitaire de l'hôte en tant qu'immunogènes (production d'anticorps per. se), haptènes (nécessitant le concours d'un haut polymère pour induire la formation d'anticorps) et immunomodulateurs. Ces derniers peuvent modifier la réponse immunitaire développée contre un antigène quelconque, soit en sens positif (immunostimulants), soit en sens négatif (immunodépresseurs). Il est bien connu que le mélange obtenu par l'inclusion de bactéries du groupe CMN dans une émulsion huile-eau (" adjuvant complet de Freund ") stimule de façon aspécifique une réponse immunitaire contre un antigène quelconque. Le principe actif de l'adjuvant de Freund est un fragment du monomère composant le peptidoglycane des organismes CMN : il s'agit du muramyl dipeptide [18].

La présence de glycolipides phénoliques chez les mycobactéries est un fait bien établi : ces composés sont immunologiquement actifs et parfois doués d'une haute spécificité d'espèce [19]. Les glycolipides phénoliques (GP), récemment découverts chez $M$. leprae, sont particulièrement importants. Des tests immunologiques, basés sur l'emploi de GP-I pour la mise en

\footnotetext{
- Species nova $=$ nouvelle espece proposée, mais pas encore approuvée par la commission internationale de taxonomie, ni publiée dans l'Int J Syst Bacter.
} 


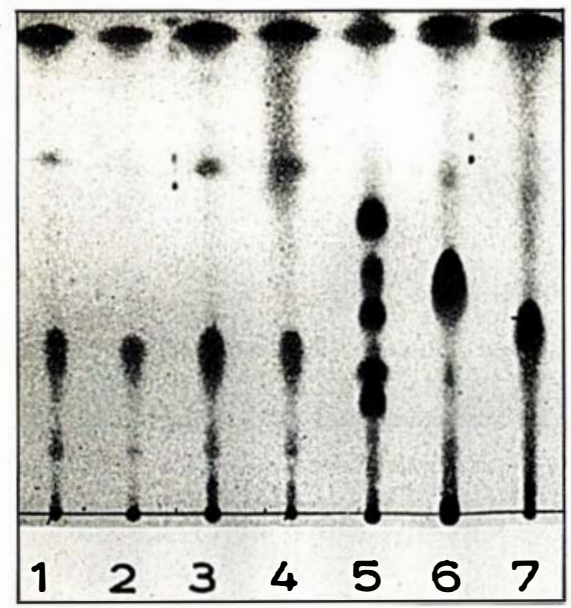

Figure 4. Acides mycoliques des micro-organismes du groupe CMN.

Les lysats méthanoliques bactériens ont été fractionnés par chromatographie en couche mince. Microorganismes: différentes souches de LDC (leprosy-derived corynebacteria) (1, 2, 3, 4), Mycobacterium avium (5). Nocardia asteroides (6), Corynebacterium xerosis (7). Notons la présence d'un seul type de composants chez les nocardias et les corynébactéries, et de trois types chez les mycobactéries de référence. [16].

évidence dans le sérum de patients d'anticorps dirigés contre $M$. leprae, ont été développés [20]. L'accumulation des glycolipides phénoliques dans les stades avancés de la maladie représente un des facteurs inhibant la stimulation des lymphocytes $\mathrm{T}$ dans la lèpre lépromateuse [3].

Le fractionnement des antigènes solubles des bactéries du groupe $\mathrm{CMN}$ est un problème majeur en chimie microbienne. Le nombre de composants à séparer et à identifier en explique la difficulté. Le développement de l'immunoélectrophorèse bidimensionnelle (figure 5) a été un événement d'importance exceptionnelle dans ce domaine [21]. Avec cette technique, l'échantillon de cytoplasme microbien, qui est fractionné par électrophorèse en une dimension, migre, en direction perpendiculaire, dans un gel renfermant un antisérum dirigé contre l'homogénat bactérien total : la réaction de chaque antigène avec l'anticorps correspondant produit une ligne $m / s n^{\circ} 8$ vol. 3 , octobre 87

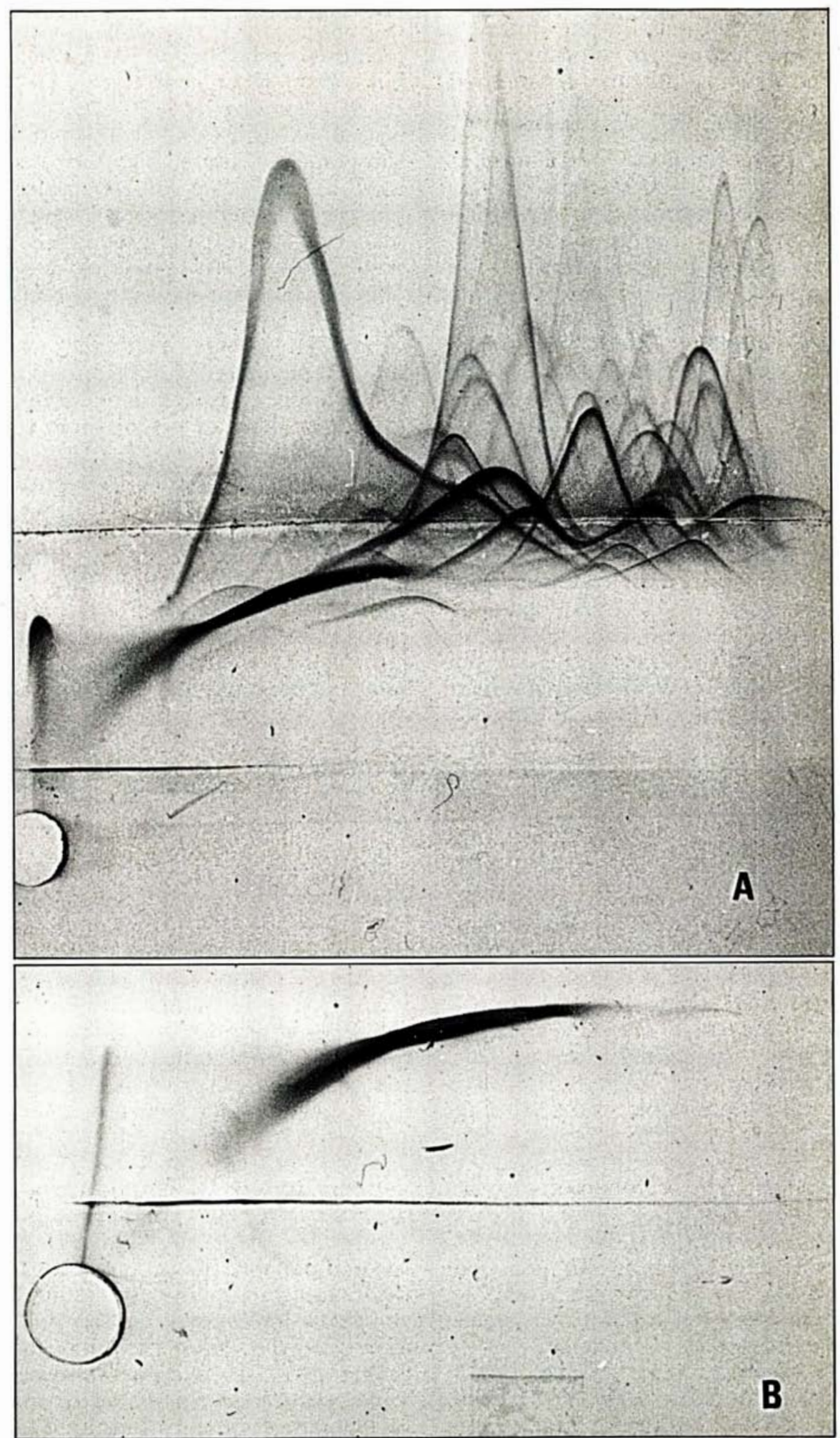

Figure 5. Fractionnement des antigènes cytoplasmiques mycobactériens. Le cytoplasme de $M$. bovis a été fractionné par immunoélectrophorèse bidimensionnelle. Migration en $1^{\text {re }}$ dimension vers la droite, et en $2^{\circ}$ dimension vers le haut, en présence d'antisérum : les immunoprécipités sont colorés au bleu de Coomassie (A). L'antigène A60, membre de la famille TMA (thermostable macromolecular antigens), a été purifié et soumis au même procédé (B). [22]. 
d'immunoprécipitation mise en évidence par un colorant.

La figure 5 illustre la méthode développée dans le laboratoire du Pr. M. Harboe à l'Université d'Oslo, méthode permettant la séparation de plusieurs dizaines d'antigènes qui sont répertoriés selon un système de référence [21]. Le composant se trouvant le plus proche du point d'application correspond à l'antigène $60(\mathrm{~A} 60)$ de $M$. bovis (figure 5) ou à l'antigène 7 (A7) de $M$. leprae, qui sont les composants majeurs de la tuberculine et de la lépromine, respectivement. Ces deux réactifs, employés - comme nous l'avons évoqué - depuis environ un siècle dans le diagnostic et le pronostic de la tuberculose et de la lèpre, sont susceptibles d'induire des réactions d'hypersensibilité retardée, expression de l'immunité cellulaire. En réalité, l'A60 et l'A7 sont les membres les plus connus d'une famille d'antigènes dits TMA (thermostable macromolecular antigens) que l'on retrouve probablement dans toutes les bactéries du groupe CMN. L'appellation sus-dite met en évidence les deux propriétés majeures de ces antigènes : la thermostabilité et le poids moléculaire élevé. La composition de l'A60 a été décrite (il s'agit d'un complexe lipo-polysaccharide-protéine) [22] et l'on pense que les antigènes TMA des autres organismes CMN ont une architecture moléculaire semblable. L'A60 a été récemment employé comme réactif de base dans un test immunologique de la tuberculose. Des essais analogues, basés sur les autres antigènes du groupe TMA, pourraient être développés pour les autres organismes CMN et plus spécialement pour les bactéries associées à la lèpre.

L'existence d'une parenté immunologique entre LDC et $M$. leprae est connue depuis de nombreuses années, les anticorps contenus dans le sérum de lépreux étant capables de reconnaître aussi bien $M$. leprae que les LDC [23]. On sait à présent que cela est largement dû à une réactivité croisée et l'antigène $M_{1}$ des LDC, deux membres de la famille TMA [24]. Un certain synergisme entre LDC et $M$. leprae a été observé au niveau expérimental. L'injection simultanée d'une faible dose de LDC vivants et de $M$. leprae dans une patte de souris et de $M$. leprae seul dans l'autre patte produit une prolifération de $M$. leprae vingt fois plus élevée dans la patte ayant reçu le mélange de LDC et de $M$. leprae [25]. Cette expérience ne prouve pas pour autant que la coopération entre les deux organismes ait lieu au niveau immunologique.

Les développements les plus récents dans le domaine sont : les anticorps monoclonaux et les travaux de génie génétique. Des anticorps monoclonaux dirigés contre $M$. leprae ont ainsi été préparés dans plusieurs laboratoires $[26,27]$. Ces anticorps ont permis, notamment, la mise en évidence d'épitopes spécifiques à $M$. leprae, lors du fractionnement électrophorétique du cytoplasme microbien. Leur application dans le diagnostic de la lèpre est en cours.

D'autre part, l'obtention d'antigènes mycobactériens pour le développement de tests immunologiques a été empêchée par l'impossibilité de cultiver $M$. leprae in vitro. Pour contourner cet obstacle, la production d'antigènes (ou de fragments d'antigènes renfermant des épitopes spécifiques) par génie génétique a été envisagée. Ainsi, des segments du génome de $M$. leprae ont été clonés dans des vecteurs d'expression qui, introduits dans $E$. coli, ont permis la production par les bactéries recombinantes de polypeptides reconnaissant les anticorps monoclonaux développés contre $M$. leprae [28, 29]. Ces travaux, en progression dans plusieurs laboratoires, pourraient aboutir au développement de dosages immunologiques spécifiques, d'exécution facile. Ils seraient à la base d'études épidémiologiques dans les pays où la lèpre est endémique et constitueraient un premier pas vers l'éradication de cette maladie aussi vieille que l'homme

\section{Summary}

This review focuses on three kinds of leprosy-associated microorganisms : Mycobacterium leprae or ML (an acid-fast bacterium not multiplying axenically), LDC or leprosy-derived corynebacteria isolated from patients' granulomas and blood, and ADM or armadilloderived mycobacteria. $M$. leprae has a peptidoglycan with glycine in place or L-alanine, a DNA with $56 \%$ GC, and a peculiar mycolic acid pattern. LDC are true corynebacteria (presence of corynomycolic acids and $56 \%$ GC in DNA) with a special DNA restriction pattern (fully A-methylated GATC sequences) and some unusual traits of the cell wall components. Contrasting with the genetic homogeneity of ML and LDC, is the genetic heterogeneity of ADM (62 to $68 \% \mathrm{GC}$, the range of genus Mycobacterium) : the five ADM subgroups behave as distinct mycobacterial species. Putative implication of LDC in leprosy is based on : a) the repeated isolation of these organisms from leprosy granulomas (but not from normal skin) ; b) the genetic homogeneity of the group ; and c) the facilitation of $M$. leprae growth produced in experimental animals by LDC. An immunological kinship of ML and LDC (crossreactivity between $\mathrm{ML}$ antigen A7 and LDC antigen $M_{1}, 2$ members of the TMA antigen family) has been observed.

\section{Remerciements}

Les recherches des auteurs dans le domaine de la lèpre ont été soutenues par des subsides octroyés par l'Association "Les Amis du Père Damien "(Bruxelles).

\section{TIRÉS A PART}

Carlo Cocito : unité de microbiologie générale et de génétique moléculaire, ICP, université de Louvain, école de médecine, avenue Hippocrate 75, B-1200, Bruxelles, Belgique. 